\title{
Hipertermia posterior al uso de misoprostol sublingual en el manejo de atonía uterina
}

\author{
DOI: https://doi.org/10.5377/alerta.v3i1.8959
}

\section{Ronald Edgardo López Guevara ${ }^{1^{*}}$}

1. Doctor en medicina, especialidad en ginecología y obstetricia, subespecialidad en obstetricia crítica, jefe de la Unidad de pacientes críticos, Hospital Nacional de la Mujer “Dra. María Isabel Rodríguez”, San Salvador, El Salvador.

${ }^{*}$ Correspondencia

$\square$ edgardo112@hotmail.com

(1) 0000-0003-1668-2280

Resumen

La hipertermia secundaria al uso de misoprostol por la vía sublingual ya ha sido reportada en la literatura; en algunos casos requiere manejo en unidades de cuidados intensivos. Se presenta el caso de una paciente con hemorragia post parto por atonía uterina, quien fue tratada con misoprostol sublingual de 800 microgramos, posteriormente la paciente presentó hiperpirexia, como parte de la evolución clínica y el manejo recibido. La paciente tuvo una buena evolución clínica.

\section{Palabras clave}

Misoprostol, hipertermia, hemorragia posparto.

\begin{abstract}
Hyperthermia secondary to the use of sublingual misoprostol is reported in the literature; in some cases, it requires management in intensive care units. We present the case of a patient with postpartum hemorrhage due to uterine atony, who receives an 800 micrograms dose of sublingual misoprostol, subsequently presenting hyperpyrexia, as part of her clinical evolution and management. Currently, the International Federation of Gynecology and Obstetrics recommends an 800 micrograms sublingual dose schedule. A patient with hyperthermia associated with the use of sublingual misoprostol is presented, with good clinical evolution.
\end{abstract}

Keywords

Misoprostol, hyperthermia, postpartum hemorrhage.

\section{Introducción}

El misoprostol es un medicamento ampliamente utilizado en la obstetricia, en especial en el manejo de hemorragia posparto (HPP), la cual sigue siendo una de las principales causas de muerte obstétrica y prevenible en todo el mundo. Este medicamento no está exento de efectos secundarios. En este caso, el misoprostol ha sido asociado a fiebre en el $44 \%$ de los casos $^{1-4}$. Existen casos tan severos con importantes cambios hemodinámicos y neurológicos que ameritan manejo en cuidados intensivos. El siguiente caso clínico aporta información sobre el cuadro clínico, resultado de exámenes de laboratorio y evolución de un caso de HPP manejada con misoprostol vía sublingual con las dosis recomendadas por la Federación Internacional de Ginecología y Obstetricia, 600 mcg como prevención y 800 mcg vía sublingual como tratamiento; con el objetivo de conocer posibles efectos secundarios importantes como la hipertermia y su manejo.
Hyperthermia after the use of sublingual misoprostol in the management of uterine atony

Citación recomendada:

López Guevara RE. Hipertermia posterior al uso de misoprostol sublingual en el manejo de atonía uterina. Alerta 2020; 3(1):9-12. DOI: https://doi. org/10.5377/alerta.v3i1.8959

Recibido:

12 de diciembre de 2019

Aceptado:

10 de enero de 2020

Publicado:

27 de enero de 2020

Contribución de autoría:

RELG': Diseño y elaboración de manuscrito.

\section{Conflicto de intereses:} El autor declara no tener conflictos de intereses. 


\section{Presentación del caso}

Paciente de 29 años con embarazo de 42 semanas de edad gestacional, quien llevó control prenatal sin anormalidad. La paciente fue referida de un hospital de segundo nivel al Hospital Nacional de la Mujer "Dra. María Isabel Rodríguez" y fue atendida en el servicio de emergencia por embarazo prolongado y oligoamnios severo. La paciente se presentó en aparente buen estado de salud y con los siguientes signos vitales: presión arterial de 100/60 mmHg, frecuencia cardiaca de 76 latidos por minuto, frecuencia cardiaca fetal de 134 latidos por minuto e índice de masa corporal de 30. En la evaluación obstétrica se identificó una altura uterina de de $30 \mathrm{~cm}$, una presentación fetal cefálica, dilatación cervical de $1 \mathrm{~cm}$ con borramiento del $20 \%$.

Debido al deterioro fetal se desarrolló una cesárea bajo anestesia raquídea, obteniendo un feto de $3 \mathrm{~kg}$ con APGAR de 9-9. Se observó poca cantidad de líquido teñido de meconio. Durante la cirugía se presentó una atonía uterina con pérdida sanguínea estimada de $1200 \mathrm{ml}$, por lo cual la paciente fue tratada con oxitocina y masaje uterino bimanual.

Hora y media después presentó un nuevo episodio de atonía. La paciente se encontraba consciente, con mucosas secas, útero atónico para 22 semanas, con presión arterial de 120/80 mmHg, frecuencia cardiaca de 98 latidos por minuto, iniciando inmediatamente el manejo médico a través de masaje uterino bimanual (extrayéndose $500 \mathrm{ml}$ de sangre y coágulos), administración de metilergonovina $0.2 \mathrm{~g}$ por vía endovenosa (repitiendo la dosis 15 minutos después) y pos- teriormente misoprostol de 800 mg por vía sublingual, logrando controlar el cuadro de atonía uterina. Una hora después la paciente presentó hipertermia $\left(42^{\circ} \mathrm{C}\right)$, frecuencia cardíaca de 192 latidos por minuto y deterioro del estado neurológico, por lo que se trasladó a la unidad de pacientes críticos, encontrándose con una presión arterial de 119/64 mmHg, frecuencia cardiaca de 119 latidos por minuto, saturación de oxígeno del $98 \%$, temperatura de $41{ }^{\circ} \mathrm{C}$, frecuencia respiratoria de 28 ciclos por minuto, Glasgow Coma Score de 7 puntos, útero contraído para 18 semanas, loquios de color y olor normal y llenado capilar de 3 segundos.

Debido al deterioro del estado neurológico se realizó intubación oro traqueal y se inició el soporte con ventilación mecánica por 24 horas, así como el manejo con antipiréticos y medios físicos para disminuir la hipertermia. Se indicó hemograma, pruebas de función renal, pruebas de coagulación y perfil hepático y electrolitos, obteniendo resultados normales en todas las pruebas.

La paciente evolucionó hasta lograr una estabilidad hemodinámica. La hipertermia cedió con los antipiréticos orales y medios físicos (Figura 1). El estado de conciencia se recuperó progresivamente sin necesidad de proporcionar soporte inotrópico o vasopresor. De esta misma forma se retiró paulatinamente la ventilación hasta conseguir respiración espontánea. Se verificó en el expediente clínico que no se administraron otros fármacos a la paciente. Finalmente, la paciente fue trasladad 48 horas después de la Unidad de Pacientes Críticos a servicio de hospitalización estable. No se registraron secuelas en órganos blancos (Tabla 1).

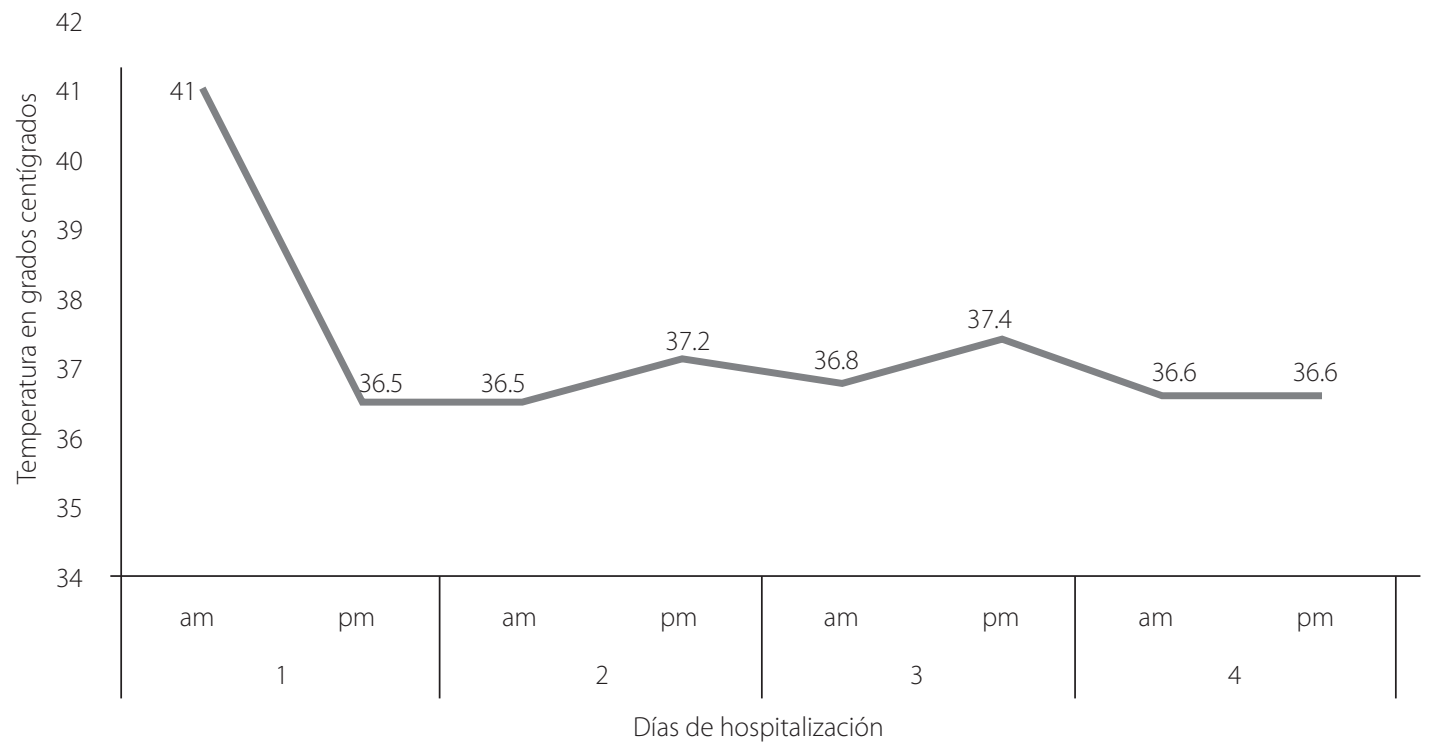

Figura 1. Gráfico de Curva de Temperatura de Paciente 
Tabla 1. Exámenes de laboratorio durante estancia en Unidad de Pacientes Críticos

\begin{tabular}{lrrr}
\hline & \multicolumn{1}{c}{ Día 1 } & \multicolumn{2}{c}{ Día 2 } \\
\hline Hemóa 3 obina (g/dL) & 12.2 & 12.5 & 10.6 \\
Hematocrito (\%) & 35.7 & 36.7 & 30.6 \\
Leucocitos x mm3 & 17,070 & 13,580 & 13,910 \\
Neutrofilos (\%) & 78.6 & 76.7 & 83.3 \\
Linfocitos (\%) & 13.6 & 15.5 & 12.7 \\
Plaquetas x mm3 & 138,000 & 93,000 & 114,000 \\
Proteína C Reactiva & 5.7 & 90 & 190 \\
(mg/Lt) & & & \\
Creatinina serica & 0.84 & 0.99 & 0.73 \\
(mg/dL) & & & \\
Glicemia (mg/dL) & 80 & N/D & 77 \\
Bilirrubina total (mg/ & 1.29 & 1.89 & 0.88 \\
dL) & & & \\
Sodio (mEq/Lt) & 140 & 142 & 141 \\
Potasio (mEq/Lt) & 4.5 & 4.4 & 3.6 \\
LDH (UI) & 538 & 834 & 386 \\
Fibrinógeno (mg/dL) & 262 & 307 & 518 \\
TP (segundos) & 9.6 & 9.3 & 9.5 \\
TPT (segundos) & 29.2 & 35.1 & 35.8 \\
\hline Fuente: Sistema de Laboratorio en Línea (SIRLAB) & &
\end{tabular}

\section{Discusión}

El misoprostol presenta efectos secundarios de manera frecuente. Entre los más comunes es la fiebre y en algunos casos la hipertermia hasta en un 35\%, como lo demostró Durocher en un estudio de cohorte en Ecuador, cuya presentación suele ser a las 1.5 horas de su administración y la cual suele declinar en las primeras 3 horas 4 .

Por lo general, las dosis recomendadas por la Federación Internacional de Ginecología y Obstetricia y la Organización Mundial de la Salud (OMS) para el uso de misoprostol como profilaxis de HPP por la vía sublingual, son de 600 mcg, asociadas siempre a fiebre y escalofríos. Las dosis de misoprostol como terapéutica por la vía sublingual son de 800 mcg, asociadas a mayor hipertermia e ingreso a cuidados intensivos ${ }^{5-6}$.

La vía sublingual asegura una concentración máxima más alta con efectos clínicos más rápidos y por ello es más común ver efectos secundarios al utilizarlos de esta manera. En el caso de emergencias obstétricas como la HPP se busca un efecto rápido, lo cual hace esta vía muy útil?.

La evolución de los pacientes suele ser a la normalización, sin ameritar medicamentos especiales y sin repercusión hemodinámica ni en otros órganos, por lo que su manejo suele ser de sostén o soporte y control de hiperpirexia ${ }^{8,9}$. En el contexto del uso de dosis de misoprostol para el tratamiento de
HPP, la fiebre e hiperpirexia son atribuibles a dicho fármaco, ya que no hay circunstancias clínicas que presenten un cuadro similar. Sin embargo, ante cualquier mujer embarazada o puérpera se deben descartar causas infecciosas, particularmente corioamnionitis. En este caso se descartó cuadro infeccioso.

Es importante que se apliquen las dosis recomendadas por la Federación Internacional de Ginecología y Obstetricia, para la vía sublingual de no más de 600 microgramos, para evitar la presencia de hiperpirexia e ingresos a cuidados intensivos por este motivo. En el caso de presentarse este cuadro en instituciones que no cuenten con unidades de pacientes críticos o unidades de cuidados intensivos o intermedios, se recomienda siempre el manejo de sostén, asegurar la vía aérea si el Score de Glasgow menor a 8, con intubación orotraqueal y referir al hospital que cuente con dichas instalaciones para su manejo.

Cuando se presenten estos casos, siempre es importante tener en cuenta que la evolución es de corta duración para la hiperpirexia y que al pasar la vida media del mismo medicamento ceden todos los efectos, ameritando únicamente manejo de soporte.

Al momento no hay estudios publicados a nivel nacional sobre la incidencia de efectos secundarios asociados a misoprostol, lo cual abre una línea de investigación.

\section{Conclusiones}

El uso de misoprostol para el manejo de la HPP por atonía uterina podría favorecer el aparecimiento de fiebre; sin embargo, es importante que se descarte otras posibles causas de la hipertermia.

\section{Aspectos éticos}

No se violentó la confidencialidad del paciente.

\section{Referencias bibliográficas}

1. Winikoff B, Dabash R, Durocher J, Darwish E, Nguyen TNN, León W, et al. Treatment of post-partum haemorrhage with sublingual misoprostol versus oxytocin in women not exposed to oxytocin during labour: a double-blind, randomised, non-inferiority trial. Lancet Lond Engl. 2010; 375(9710):2106. DOl: https://doi.org/10.1016/s01406736(09)61924-3

2. Martínez Pacheco FH, Zambrano Benítez CP. Reporte de caso y revisión de la literatura: 
hiperpirexia como efecto secundario posterior a la administración de misoprostol por vía sublingual en el manejo de la hemorragia posparto. Rev Colomb Anestesiol. 2013; 41(1):65-8. DOI: https://doi. org/10.1016/j.rca.2012.08.004

3. Blum J, Winikoff B, Raghavan S, Dabash R, Ramadan MC, Dilbaz B, et al. Treatment of post-partum haemorrhage with sublingual misoprostol versus oxytocin in women receiving prophylactic oxytocin: a doubleblind, randomised, non-inferiority trial. The Lancet. 2010; 375(9710):217-23. DOI: https:// doi.org/10.1016/s0140-6736(09)61923-1

4. Durocher J, Bynum J, León W, Barrera G, Winikoff B. High fever following postpartum administration of sublingual misoprostol. BJOG Int J Obstet Gynaecol. 2010;

117(7):845-52. DOl: https://doi.org/10.1111/ j.1471-0528.2010.02564.x

5. WHO. WHO recommendations for the prevention and treatment of postpartum haemorrhage [Internet]. WHO. [citado 9 de enero de 2020]. Disponible en: https://bit. Iy/3aXxB7A
6. Morris JL, Winikoff B, Dabash R, Weeks A, Faundes A, Gemzell-Danielsson K, et al. Recomendaciones actualizadas de figo para el uso de misoprostol solo en ginecología y obstetricia. FASGO 2017. Disponible en: https://bit.ly/37IVKgd

7. Tang, O, GemzellखDanielsson, K. and Ho, P. (2007), Misoprostol: Pharmacokinetic profiles, effects on the uterus and side区 effects. International Journal of Gynecology \& Obstetrics. 2017; 99:S160-S167. DOI: https://doi.org/10.1016/j.ijgo.2007.09.004

8. León W, Durocher J, Barrera G, Pinto E, Winikoff B. Dose and side effects of sublingual misoprostol for treatment of postpartum hemorrhage: what difference do they make? BMC Pregnancy Childbirth. 2012; 12:65. DOl: https://doi. org/10.1186/1471-2393-12-65

9. Tolefac PN, Minkande JZ. Sublingual misoprostol and hyperpyrexia: case report with temperature curve. BMC Research Notes. 2017; 10 (1): 329. DOI: https://doi. org/10.1186/s13104-017-2661-2 